\title{
A Leaky Coaxial Cable Antenna based on Sinusoidally-Modulated Reactance Surface
}

\author{
Zeeshan Siddiqui ${ }^{(1)}$, Ahmed Radwan ${ }^{(1)}$, Marko Sonkki ${ }^{(1)}$, Marko Tuhkala ${ }^{(2)}$, Sami Myllymäki ${ }^{(2)}$
}

(1) Centre for Wireless Communications (CWC), University of Oulu, Finland.

(2) Microelectronics Research Unit, University of Oulu, Finland.

\begin{abstract}
This paper presents the simulated results of a leaky coaxial cable antenna (LCXA) based on sinusoidally modulated reactance surface (SMRS). The theory of SMRS is employed to transform the leaky coaxial cable (LCX), a uniformly radiating structure into a directional antenna, LCXA. It is shown that high gain and arbitrary direction of radiation could be achieved by LCXA.
\end{abstract}

Keywords - indoor wireless systems, leaky coaxial cable, sinusoidally modulated reactance surface

\section{INTRODUCTION}

Leaky coaxial cable (LCX) or the leaky feeder is being utilized in mines and tunnels communication systems for around half century. However, there is a renewed interest in finding new applications for the LCX like, utility in indoor communication systems, inflight wireless communication systems and sensors integration [1] [2] [3]. In its conventional application site, mines and tunnels, the uniformly radiating nature is an advantage over the conventional antennas but in different scenario like buildings and warehouses, it lacks the ability to sufficiently cover the space. It is due to the hybrid spatial nature of the indoor places, mix with long corridors, halls and rooms.

In this communication, we present a novel LCXA design based on the sinusoidally modulated reactance surface (SMRS). The application of the SMRS theory transforms the radiation characteristics of the LCX. An arbitrary direction of radiation could be achieved through a judicious design of the reactance surface. The modulated reactance is achieved by periodically varying the length of the slots across the cable as show later in the paper.

To improve the radio coverage at indoor places, we propose a solution to hybrid the LCX with LCXA sections in order to have directional radiation at required locations.

\section{THEORETICAL BACKGROUND}

\section{A. Leaky Coaxial Cable}

Leaky coaxial cable (LCX) is a coaxial cable with purposely made holes or slots on its outer conductor. The wave leaks or radiates from the slots as it propagates inside the cable. LCX is broadly classified in two types depending on the slots shape and spacing as coupled mode and radiating mode cable as shown in figure. Here we consider a coupled mode cable, it is well known that two distinct modes namely, biflar and monofilar are supported by the coupled mode LCX. The bifilar mode is mainly confined between the center and outer conductor of the coaxial cable while some of the energy leaks outside from the slots, which is generally utilized for the communication purpose. On the contrary, the monofilar mode is spread over the outer side of the outer conductor similar to surface wave with some leakage inside. The speed of monofilar mode is slightly slower than the wave in free space and the coupled mode LCX is generally categorized as slow wave structure [4]. 


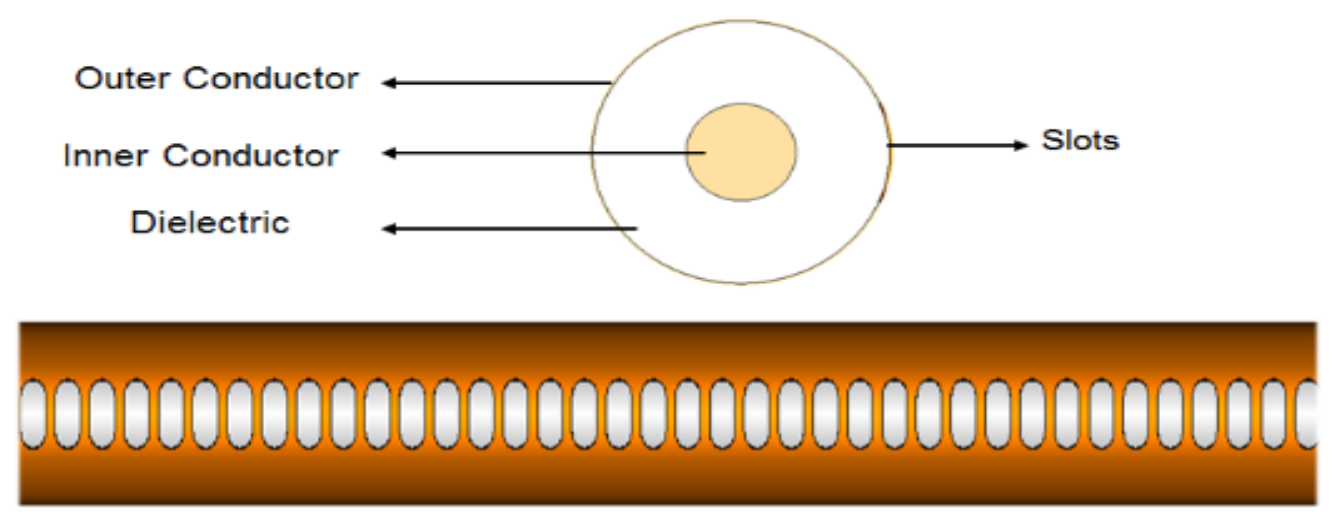

Fig. 1. A coupled mode leaky coaxial cable

\section{B. Sinusoidally modulated reactance surface}

SMRS refers to any plane surface whose modal surface impedance is sinusoidally modulated. The propagation characteristics and relevance of the SMRS to high gain modulated surface wave antenna was theoretically investigated by A. Oliner and Hessel [5]. They showed that, by periodically modulating the surface reactance above a threshold period, single or multiple discrete modes of the surface wave become propagating. In this way, power is radiated away from the surface wave. The surface impedance, $\eta$ of a SMRS in xy-plane with the direction of wave propagation assumed to be in z-direction, is given by following equation [5].

$$
\eta(z)=j \eta_{o} X\left[1+M \cos \left(\frac{2 \pi z}{a}\right)\right]
$$

where $\eta_{o}$ is free-space wave impedance, $X$ is the average surface reactance normalized by free-space wave impedance, $M$ is the modulation factor, $a$ is the periodicity of modulation. It is basically $(X, M, a)$ which defines the characteristics of a SMRS.

\section{DESIGN AND SIMULATION SETUP}

First we designed and simulated a coupled mode LCX. The numerical simulations are carried out by using commercial CST Microwave Studio. The opted design for the LCX has closely spaced slots. The slots distance is very small relative to the operating wavelength. We exclude the outer jacket from the simulation model as it has minimal effect on the electrical performance of the cable. The characteristic impedance is designed to be $50 \mathrm{ohm}$, the inner and outer conductor diameter are 9.8 and $25 \mathrm{~mm}$ respectively. The dielectric constant for the foam confined between the conductors is 1.4. The effect of slot length is studied, the field strength increase with the increase in the slot length to a certain value. The simulated length of the cable is around $1 \mathrm{~m}$ and there are normal coaxial cable sections at both the ends. The length of simulated leaky cable section is $959.5 \mathrm{~mm}$. 


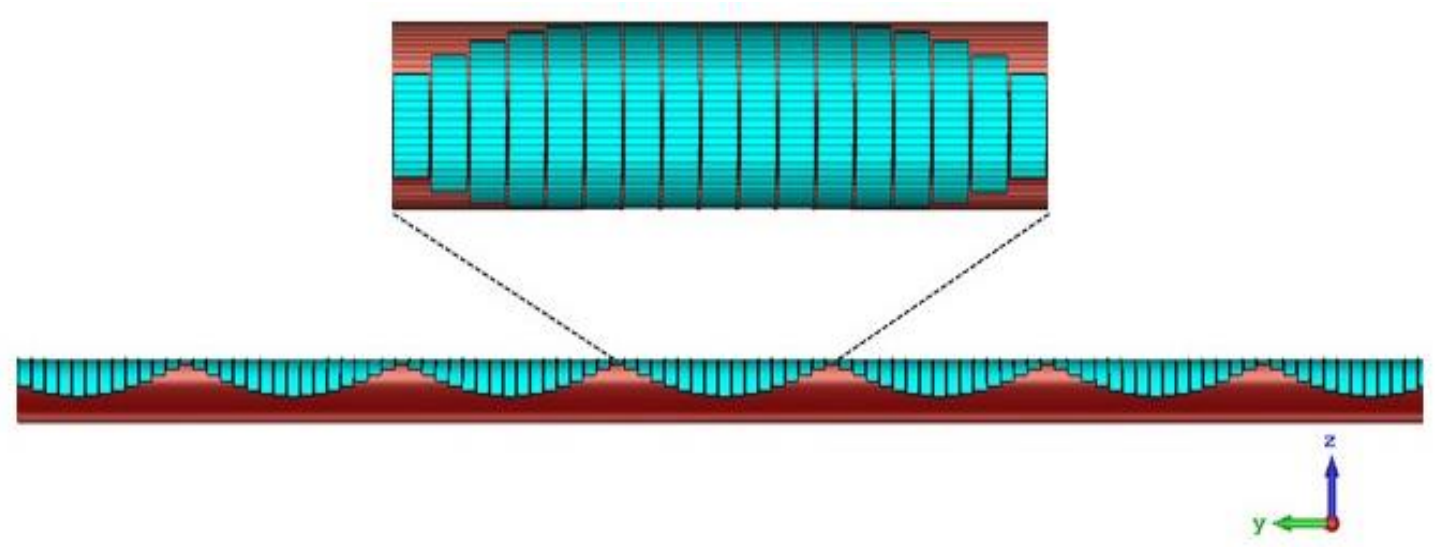

Fig. 2. Simulation model for the leaky coaxial cable antenna

For the application of SMRS theory, we choose the design frequency to be $2.4 \mathrm{GHz}$. The desired direction of maximum radiation is $10^{\circ}$. As discussed above, application of the SMRS theory basically requires the design of $(X, M, a)$. In this communication, we are not strictly relying on the SMRS theory rather a combination of it with full wave simulations. The nature of radiation is complex here, the bifilar mode leakage is already in action while the surface wave mode, monofilar mode, is also transforming into radiating mode through the SMRS application. The propagating surface wave mode is hybrid TE and TM. Also from theoretical point of view, there is a freedom of designing average surface reactance, $X$, but from practical implementation aspect, the surface reactance could be varied around a certain value depending on the selected structure.

Initiating the design by selecting an appropriate periodicity, a mono-harmonic radiation is desired. Generally the condition is satisfied with $\lambda_{0} / 2<a<\lambda_{0}$, where $\lambda_{0}$ is the free-space wavelength. The wave length is $125 \mathrm{~mm}$ at $2.4 \mathrm{GHz}$, we selected $a$ to be around $0.75 \lambda_{0}$. According to theory [6], the next step is to calculate the average surface reactance from the chosen periodicity, here we relied on the simulations and found the appropriate values through iterative simulations. In this study the slot length is varied from $15 \mathrm{~mm}$ to $45 \mathrm{~mm}$. The slot width is fixed at $5.5 \mathrm{~mm}$ and the spacing between the slots is kept at $0.25 \mathrm{~mm}$. The theory specifies that $X$ and $a$ dictates the beam direction while $M$ controls the leakage rate or the radiation efficiency. We simulated ten periods along the length of the cable. The simulation model is shown in Fig. 2.

\section{RESULTS DISCUSSION}

First we consider the impedance matching, to observe the effect of SMRS application. S11 plot is shown in Fig. 3, there is a hump around $2.2 \mathrm{GHz}$ crossing $-10 \mathrm{~dB}$ limit. This is due to directed beam is at true broadside causing high reflection. There are different techniques discussed in the literature to compensate the cause [7]. Other than the frequencies with beam at broadside the antenna is well matched. The 3D realized gain plots are shown in Fig. 4. The simulate beam directions are according to the design. The realized gain is satisfactory at a level of above $10 \mathrm{~dB}$. The side lobes strength need to be further decreased. Study is in-progress to improve the impedance matching and side lobe performance of the antenna. 


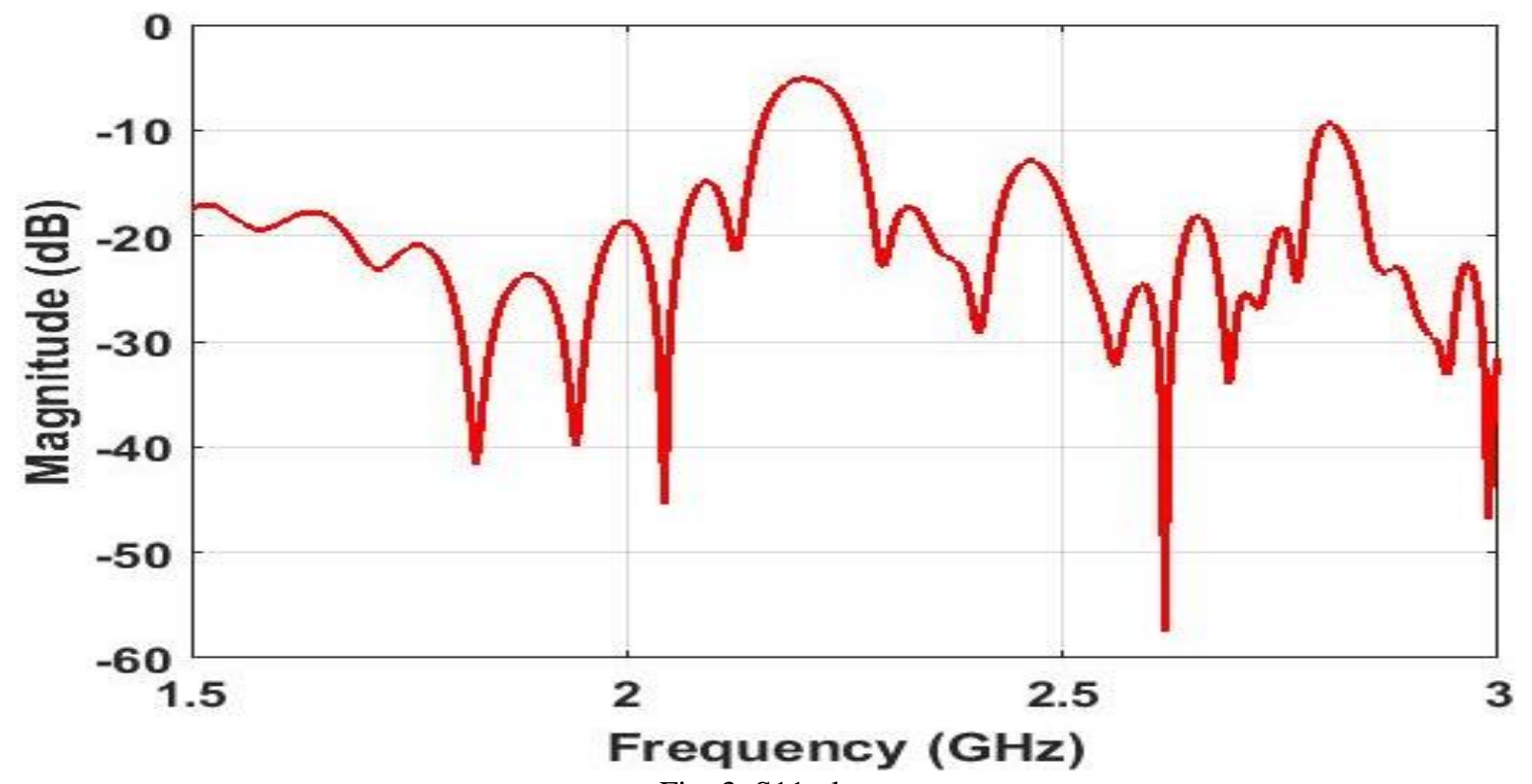

Fig. 3. S11 plot
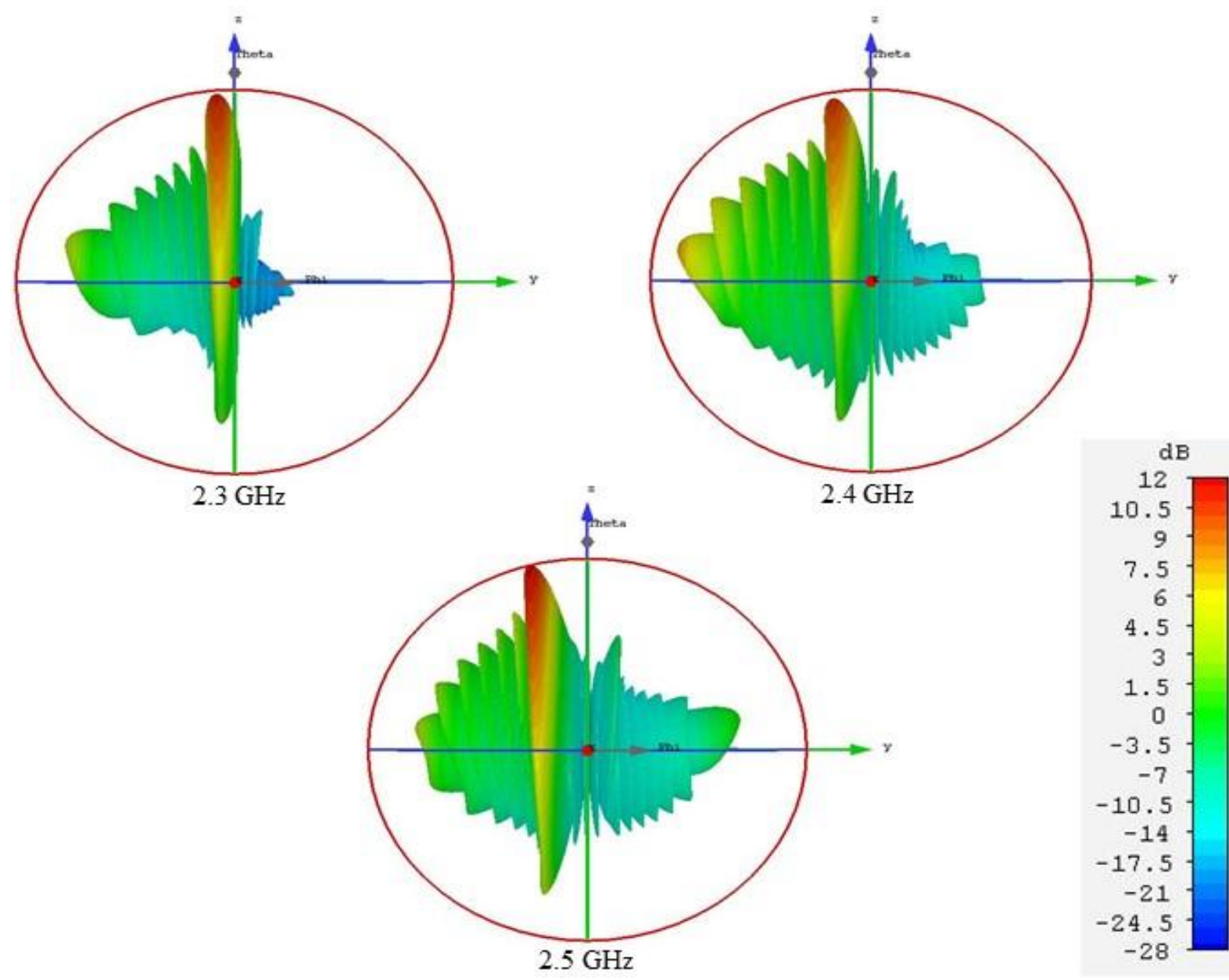

Fig. 4. 3D plots, realized gain 


\section{CONCLUSION}

In this paper, we present a novel leaky coaxial cable antenna (LCXA) based on sinusoidally modulated reactance surface (SMRS). By designing the modulation period, modulation index and average reactance, tightly bounded surface wave of the monofilar mode on the outer conductor of the LCX are transformed into a radiating leaky wave at an arbitrary direction. In the regular coupled mode LCX operation the leaked energy is only sufficient for communication coverage within few meters. It is designed and simulated here that by sinusoidally varying the slot length much higher radiation efficiency and gain could be achieved with the same length of cable.

The proposed solution may find its utility in distributed antenna systems (DAS) due to its robustness and ease of integration. Also it is possible to make a hybrid solution, LCX combined with LCXA to cover places with varying coverage requirement at different locations like rooms, halls and corridors as the leakage rate and the directivity can be controlled in comparison with the regular LCX.

\section{ACKNOWLEDGEMENT}

This work is sponsored by Finnish Funding Agency for Innovation, Tekes regarding Smart Wireless Cable-Project. We are thankful to our sponsors and the industrial collaborators, Prysmian Group, Elcoflex and Premix.

\section{REFERENCES}

[1] J. Medbo and A. Nilsson, Leaky coaxial cable MIMO performance in an indoor office environment, 2012, pp. 2061--2066.

[2] P. James and D. Hughes., "Multi-receiver communication system with distributed aperture antenna.". U.S. Patent Application No. 10/360,941., 2003.

[3] I. K., H. T., K. Sumi and K. Tanaka, "Wide-area surveillance sensor with leaky coaxial cables," SICE-ICAS International Joint Conference IEEE, 959-963 October 2006.

[4] E. E. Hassan, Field solution and propagation characteristics of monofilar-bifilar modes of axially slotted coaxial cable, vol. 37, IEEE, 1989, pp. 553--557.

[5] A. Oliner and A. Hessel, Guided waves on sinusoidally-modulated reactance surfaces, vol. 7, IEEE, 1959, pp. 201--208.

[6] A. M. Patel and A. Grbic, A printed leaky-wave antenna based on a sinusoidally-modulated reactance surface, vol. 59, IEEE, 2011, pp. 2087--2096.

[7] G. S. Kong, H. F. Ma, B. G. Cai and T. J. Cui, Continuous leaky-wave scanning using periodically modulated spoof plasmonic waveguide, vol. 6, Nature Publishing Group, 2016. 